\title{
Gamma-ray emission from SN2014J near maximum optical light ${ }^{\star}$
}

\author{
J. Isern ${ }^{1}$, P. Jean ${ }^{2,3}$, E. Bravo ${ }^{4}$, J. Knödlseder ${ }^{2,3}$, F. Lebrun ${ }^{5}$, E. Churazov ${ }^{6,7}$, R. Sunyaev ${ }^{6,7}$, A. Domingo ${ }^{8}$, C. Badenes ${ }^{9}$, \\ D. H. Hartmann ${ }^{10}$, P. Hoeflich ${ }^{11}$, M. Renaud ${ }^{12}$, S. Soldi ${ }^{5}$, N. Elias-Rosa ${ }^{13,1}$, M. Hernanz ${ }^{1}$, I. Domínguez ${ }^{14}$,
} D. García-Senz ${ }^{15}$, G. G. Lichti ${ }^{16}$, G. Vedrenne ${ }^{2,3}$, and P. Von Ballmoos ${ }^{2,3}$

1 Institut de Ciències de l'Espai (ICE-CSIC/IEEC), Campus UAB, 08193 Bellaterra, Barcelona, Spain e-mail: isern@ieec.cat

2 Université de Toulouse, UPS-OMP, IRAP, 31028 Toulouse, France

3 IRAP, 9 Av colonel Roche, BP44346, 31028 Toulouse Cedex 4, France e-mail: pierre.jean@irap.omp.eu

${ }^{4}$ E.T.S.A.V., Univ. Politècnica de Catalunya, c/Pere Serra 1-15, 08173 Sant Cugat del Valles, Spain e-mail: eduardo.bravo@upc.edu

5 APC, Univ. Paris Diderot, CNRS/IN2P3, CEA/Irfu, Obs. de Paris, Sorbonne Paris Cité, 10 rue Alice Domon et Leonie Duquet, 75205 Paris Cedex 13, France

6 Space Research Institute (IKI), Proufsouznaya 84/32, 117997 Moscow, Russia

7 Max-Planck-Institut for Astrophysics, Karl-Schwarzschild-Strasse 1, 85741 Garching, Germany

8 Centro de Astrobiología (CAB-CSIC/INTA), PO Box 78, 28691 Villanueva de la Cañada, Madrid, Spain

9 Department of Physics and Astronomy \& Pittsburgh Particle Physics, Astrophysics and Cosmology Center (PITT-PACC), University of Pittsburgh, Pittsburgh PA15260, USA

10 Department of Physics and Astronomy, Clemson University, Clemson, SC 29634, USA

11 Physics Department, Florida State University, Tallaharssee, FL32306, USA

12 Laboratoire Univers et Particules de Montpellier (LUPM), UMR 5299, Université de Montpellier II, 34095 Montpellier, France

13 INAF-Osservatorio Astronomico di Padua, Vicolo dell'Osservatorio 5, 35122 Padova, Italy

14 Universidad de Granada, Cuesta del Hospicio sn, 18071 Granada, Spain

15 Dept. Fisica, UPC, Compte d'Ugell 187, 08036 Barcelona, Spain

16 Max-Planck-Institut for Extraterrestrial Physics, Giessenbachstrasse 1, 85741 Garching, Germany

Received 11 July 2015 / Accepted 8 February 2016

\section{ABSTRACT}

\begin{abstract}
Context. The optical light curve of Type Ia supernovae (SNIa) is powered by thermalized gamma-rays produced by the decay of ${ }^{56} \mathrm{Ni}$ and ${ }^{56} \mathrm{Co}$, the main radioactive isotopes synthesized by the thermonuclear explosion of a $\mathrm{C} / \mathrm{O}$ white dwarf.

Aims. Gamma-rays escaping the ejecta can be used as a diagnostic tool for studying the characteristics of the explosion. In particular, it is expected that the analysis of the early gamma emission, near the maximum of the optical light curve, could provide information about the distribution of the radioactive elements in the debris.

Methods. The gamma data obtained from SN2014J in M 82 by the instruments on board INTEGRAL were analysed paying special attention to the effect that the detailed spectral response has on the measurements of the intensity of the lines.

Results. The $158 \mathrm{keV}$ emission of ${ }^{56} \mathrm{Ni}$ has been detected in SN2014J at $\sim 5 \sigma$ at low energy with both ISGRI and SPI around the maximum of the optical light curve. After correcting the spectral response of the detector, the fluxes in the lines suggest that, in addition to the bulk of radioactive elements buried in the central layers of the debris, there is a plume of ${ }^{56} \mathrm{Ni}$, with a significance of $\sim 3 \sigma$, moving at high velocity and receding from the observer. The mass of the plume is in the range of $\sim 0.03-0.08 M_{\odot}$.

Conclusions. No SNIa explosion model has ever predicted the mass and geometrical distribution of ${ }^{56} \mathrm{Ni}$ suggested here. According to its optical properties, SN2014J looks like a normal SNIa, so it is extremely important to discern whether it is also representative in the gamma-ray band.
\end{abstract}

Key words. supernovae: individual: SN2014J - gamma rays: stars

\section{Introduction}

Type Ia supernovae (SNIa) are the outcome of the thermonuclear explosion of a carbon/oxygen white dwarf in a close binary system. During this explosion, significant amounts of radioactive isotopes are produced, the most abundant being ${ }^{56} \mathrm{Ni}$, which

^ Based on observations with INTEGRAL, an ESA project with instruments and the science data centre funded by ESA member states (especially the PI countries: Denmark, France, Germany, Italy, Switzerland, and Spain), the Czech Republic, and Poland and with the participation of Russia and USA. decays to ${ }^{56} \mathrm{Co}\left(T_{1 / 2}=6.08\right.$ days $)$ and further to ${ }^{56} \mathrm{Fe}\left(T_{1 / 2}=\right.$ 77.24 days). These radioactive nuclei produce gamma-rays that thermalize in the ejecta and are thus ultimately responsible for the power of the luminous supernova. The most important lineproducing nuclear transitions are due to ${ }^{56} \mathrm{Ni}(158,480,750$, and $812 \mathrm{keV}$ ) and ${ }^{56} \mathrm{Co}(847$ and $1238 \mathrm{keV})$. To these photons one must add those produced by the annihilation of positrons either directly or through the formation of positronium. As ejecta expansion proceeds, matter becomes more and more transparent, and an increasing number of gamma-rays escape, thus avoiding thermalization. Therefore, gamma-rays escaping the ejecta can 
Table 1. INTEGRAL observation schedule (IJD: INTEGRAL Julian day).

\begin{tabular}{cccc}
\hline \hline Orbit & IJD start & IJD stop & Days after explosion \\
\hline 1380 & 5144.298 & 5146.956 & $16.5-19.2$ \\
1381 & 5147.289 & 5149.941 & $19.6-22.2$ \\
1382 & 5150.280 & 5152.699 & $22.6-25.0$ \\
1383 & 5153.925 & 5155.922 & $26.2-28.2$ \\
1384 & 5156.262 & 5158.486 & $28.6-30.8$ \\
1385 & 5159.254 & 5161.899 & $31.6-34.2$ \\
1386 & 5162.248 & 5162.858 & $34.4-35.2$ \\
\hline
\end{tabular}

Notes. The moment of the explosion of SN2014J, January 14.72 UT 2014, corresponds to JD 2456672.22 or IJD 5127.75.

be used as a diagnostic tool for studying the structure of the exploding star and the characteristics of the explosions (Clayton et al. 1969; Gómez-Gomar et al. 1998; Höflich et al. 1998; The \& Burrows 2014). In particular, the comparison between early and late spectra is especially illustrative since it is sensitive to the distribution of ${ }^{56} \mathrm{Ni}$ in the debris (Gómez-Gomar et al. 1998) and can thus advance our understanding of this crucial cosmological tool (Isern et al. 2011; Hillebrandt et al. 2013). The attempt to detect with INTEGRAL gamma-ray emission from SN2011fe in M101 failed because of its distance $(\sim 6.4 \mathrm{Mpc})$ and yielded a flux that was too faint (Isern et al. 2013). So far, the signatures of ${ }^{56} \mathrm{Ni}$ and ${ }^{56} \mathrm{Co}$ decay were observed in hard X-rays and gammarays only from the Type II SN1987A in The Large Magellanic Cloud (Sunyaev et al. 1987; Matz et al. 1988; Teegarden et al. 1989).

SN2014J was discovered by Fossey et al. (2014) on January 21 in M $82(d=3.5 \pm 0.3 \mathrm{Mpc})$. The moment of the explosion of SN2014J was estimated to be on January 14.72 UT 2014 (Zheng et al. 2014) or JD 2456672.22 . INTEGRAL began observing this source on January 31, 16.5 days after the explosion and ended February 18, 35.2 days after the explosion. Late time observations, 50-100 days after the explosion, were also programmed, allowing the detection of the ${ }^{56} \mathrm{Co}$ emission lines for the first time (Churazov et al. 2014a,b). The firm detection in SN2014J of the gamma-ray emission when the ${ }^{56} \mathrm{Ni}$ emission is still important (Isern et al. 2014) offers the opportunity to gain insight into the abundances and distribution of these radioactive isotopes.

\section{INTEGRAL observations}

INTEGRAL is an ESA scientific mission able to operate in gamma-rays, X-rays, and visible light (Winkler et al. 2003). It was launched on October 17, 2002 into a highly eccentric orbit with a period of three days and spent most of this time outside the radiation belts. The results presented here were obtained during orbits 1380-1386 as described in Table 1 (proposal number 1170001, public; proposal number 1140011, PI: Isern). Orbit 1387 was devoted to calibration, and orbit 1388 was affected by a giant solar flare.

The instruments on board are: i) the OMC camera, able to operate in the visible band up to a magnitude 18 (Mas-Hesse et al. 2003); it was used to obtain the light curve in the $V$ band, allowing an early estimate of the amount of ${ }^{56} \mathrm{Ni}$ needed to account for the shape of the optical light curve, as well as to predict the intensity of the ${ }^{56} \mathrm{Co}$ line at late times; ii) the X-ray monitors JEM-X, which work in the range of 3 to $35 \mathrm{keV}$ (Lund et al. 2003) and which were used to constrain the continuum emission of SN2014J in this band; and iii) the two main gamma

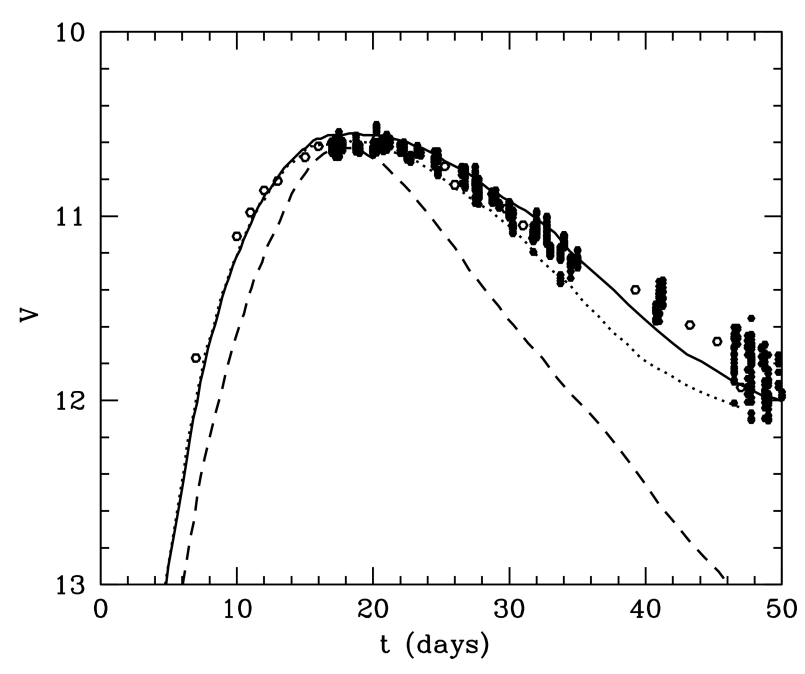

Fig. 1. Optical light curve in the $V$ band versus the time after the explosion. Filled dots represent the data obtained with the OMC camera on board INTEGRAL without correcting from extinction, empty dots the data provided by Las Cumbres Observatory Global Telescope Network (Marion et al. 2015). The solid line is a delayed detonation (DDT) that gives a good fit to the SN2014J OMC light curve. It produces $0.65 M_{\odot}$ of ${ }^{56} \mathrm{Ni}$, and ejects a total mass of $1.37 M_{\odot}$ with a kinetic energy of $1.3 \times 10^{51} \mathrm{erg}$. The dashed line represents a similar DDT explosion in which a $1.2 M_{\odot}$ white dwarf is embedded in a $0.2 M_{\odot}$ halo made of carbon and oxygen, and it is intended to be representative of merging models (double degenerates, or DD models). The dotted line is for a model fitting the light-curve properties of SN2011fe (Isern et al. 2013).

instruments, SPI, a cryogenic germanium spectrometer able to operate in the energy range of $18 \mathrm{keV}-10 \mathrm{MeV}$ (Vedrenne et al. 2003), and IBIS/ISGRI, an imager able to operate in the energy range of $15 \mathrm{keV}$ to $1 \mathrm{MeV}$ (Lebrun et al. 2003; Ubertini et al. 2003). Below $300 \mathrm{keV}$, IBIS/ISGRI is more sensitive than SPI, a factor $\sim 3$ in the region of $\sim 150 \mathrm{keV}$ (Lebrun et al. 2003; Roques et al. 2003), but the sensitivity of both instruments is good enough to allow comparison of the results in the region of 40 to $200 \mathrm{keV}$.

\subsection{The $O M C$ data}

Figure 1 displays the light curve obtained with the OMC, as well as those provided by several models obtained assuming different parameters and boundary conditions in order to illustrate the sensitivity of the light curve to different hypothesis about the origin of the supernova. The reduction of the photometric data followed the same procedures as in the case of SN2011fe (Isern et al. 2013). For SN2014J, the main difficulty is the contamination by unresolved stars in M 82 as a consequence of the large pixel size of the OMC (17.5"). This problem was overcome by subtracting images of the same region of M 82 as was obtained in 2012 .

The peak magnitude of $V=10.6$ occurred at JD $=$ $2456691.9 \pm 1,19.7$ days after the explosion, and 15 days after maximum the light curve had dropped by $0.6 \mathrm{mag}$, in agreement with observations obtained at the Las Cumbres Observatory Global Telescope Network (Marion et al. 2015). Figure 1 displays the resulting $V$-band light curve without correcting for extinction. This light curve can be compared to several theoretical models, and in principle, a matching solution can be obtained. Nevertheless, this solution is not unique since similar light curves can be obtained by conveniently tuning the different 
parameters that characterize each model family. Furthermore, environment circumstances, like the presence of circum-stellar material, can modify the shape of the light curve as can be seen in the figure. Just as an example, the model represented by a continuous line in the figure has synthesized $\sim 0.65 M_{\odot}$ of ${ }^{56} \mathrm{Ni}$.

Since the optical and infrared observations of SN2014J (Marion et al. 2015) strongly support the idea of no-mixing in the outer layers, and delayed detonation (DDT) models ${ }^{1}$ predict such behaviour, a model of this class reasonably fitting the light curve and satisfying the constraints imposed by the late observations of INTEGRAL (Churazov et al. 2014b) has been selected as a reference. This model, the DDT1P4 model, produces $0.65 M_{\odot}$ of ${ }^{56} \mathrm{Ni}$ and ejects $1.37 M_{\odot}$ of material with a kinetic energy of $1.32 \times 10^{51} \mathrm{erg}$. During the epoch corresponding to $\sim 50-100$ days after the explosion, the 847 and $1238 \mathrm{keV}{ }^{56} \mathrm{Co}$ lines obtained with this model exhibit a mean flux of $3.1 \times 10^{-4}$ and $2.2 \times 10^{-4} \mathrm{~cm}^{-2} \mathrm{~s}^{-1}$, are centred at 851 and $1244 \mathrm{keV}$, and have a FWHM of 29 and $42 \mathrm{keV}$, respectively, to be compared with the observed values $(2.34 \pm 0.7) \times 10^{-4}$ and $(2.78 \pm 0.7) \times 10^{-4} \mathrm{~cm}^{-2} \mathrm{~s}^{-1}, 852 \pm 4.5$ and $1255 \pm 7 \mathrm{keV}$, and $24 \pm 8$ and $45 \pm 14 \mathrm{keV}$ (Churazov et al. 2014b). This amount of ${ }^{56} \mathrm{Ni}$ also agrees with the value, $\sim 0.6 M_{\odot}$ obtained with the mid-infrared observations (Telesco et al. 2015).

\subsection{JEM-X data}

They were analysed with the same methods as are described in Isern et al. (2013). The flux during revolutions 1380-1386 at the position of the supernova was $1.5 \times 10^{-3} \mathrm{~cm}^{-2} \mathrm{~s}^{-1}$ in the $3-10 \mathrm{keV}$ band, while there was no detection in the $10-25 \mathrm{keV}$ range, with a $3 \sigma$ flux upper limit of $6 \times 10^{-4} \mathrm{~cm}^{-2} \mathrm{~s}^{-1}$. These fluxes are consistent with the values found in the same position before the explosion and can be attributed to the combined contribution of compact sources in M 82, in particular M 82X-1 and X-2 (Bachetti et al. 2014; Sazonov et al. 2014).

\subsection{SPI data}

The SPI data were cleaned and calibrated with the standard procedure described in Sect. 2.2 of Isern et al. (2013). During this period of observations, science windows showing high rates in the anti-coincidence system of SPI when INTEGRAL was exiting the radiation belts were removed $(\sim 2-3$ first science windows per revolution) to avoid systematic errors induced by strong background fluctuations (Jean et al. 2003, see Fig. 5).

The behaviour of the instrumental background, produced by the interactions of cosmic rays and solar protons with the instrument, is very complex (Jean et al. 2003; Weidenspointner et al. 2003). Unfortunately, the two main decay lines of ${ }^{56} \mathrm{Ni}$, the $158 \mathrm{keV}$ and $812 \mathrm{keV}$ lines, may be affected by two instrumental lines due to decays of ${ }^{47} \mathrm{Sc}$ and ${ }^{58} \mathrm{Co}$ that produce lines at $159 \mathrm{keV}$ and $811 \mathrm{keV}$, respectively, depending on the shift of the ${ }^{56} \mathrm{Ni}$ lines with respect to their canonical energy.

The spatial and temporal modulations produced by the coded mask and dithering allow the background lines to be rejected as long as their positions and widths are well aligned among the detectors and the detector pattern (the relative count rate between detectors) is well known. The procedure adopted here consists in adjusting the flux from SN2014J for each energy bin in two steps. In the first step, a background count rate was obtained

\footnotetext{
1 Models in which the flame starts at the centre and propagates subsonically, making a transition to a supersonic regime when the density is low enough (Höflich et al. 2002).
}

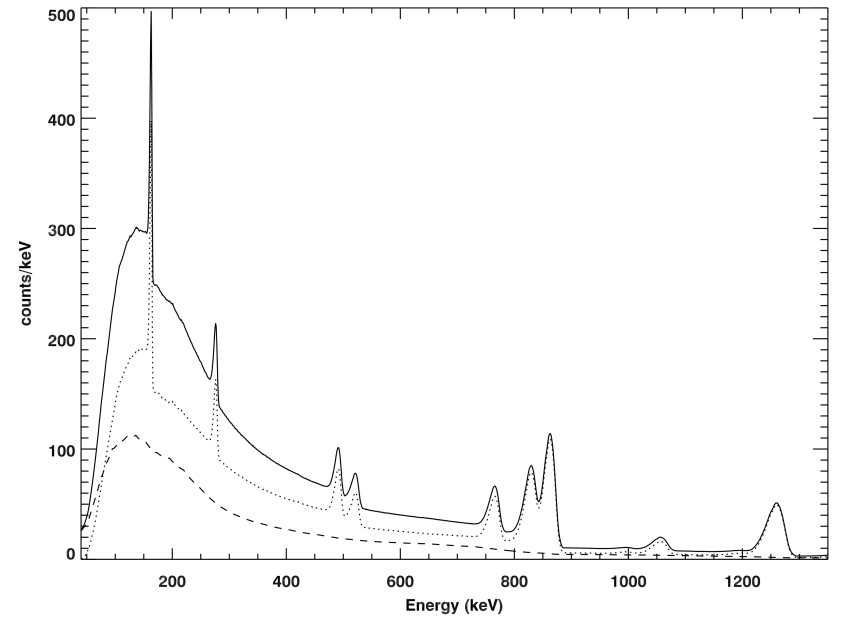

Fig. 2. SPI spectral response to the DDT1p4 model during revolutions 1380-86. The dotted and dashed lines represent the contribution of the diagonal and off-diagonal terms of the convolved spectrum, respectively. The solid line is the sum of both components. The diagonal component scales with the original model, while the non-diagonal component is produced by the energy redistribution of high energy photons and contributes to the continuum with an amount comparable to that of the diagonal component in the $100-200 \mathrm{keV}$ range.

per orbit, detector, and energy bin to fix the detector pattern. In the second step, a global background rate factor was fitted per pointing, keeping the detector pattern (i.e. the relative count rate between detectors) fixed to the values determined in the first step. Despite such precautions, some residual instrumental lines could remain, and since these lines are intrinsically narrow, any narrow feature of the observed spectrum risks being confused with them if the background is not correctly modelled.

Indeed, one of the main problems in interpreting the data is that the flux extracted in an energy bin $E_{\mathrm{i}}$ contains not only the source photons emitted at this energy (later called diagonal terms) but also those emitted with an energy $E>E_{\mathrm{i}}$ that do not deposit all their energy in the detectors (e.g. Compton edge, backscattering photons - later called off-diagonal terms). This last contribution is not negligible at low energies and can be obtained by comparing the extracted spectrum with the theoretical spectra duly convolved with the spectral response of the instrument. Therefore, to make a meaningful comparison between the spectra measured with SPI and theoretical models, they have to be convolved with the instrumental response to take the offdiagonal terms of the spectral response into account.

In the case of SPI, the convolved spectrum is calculated for a given theoretical model taking the SPI IRF (imaging response files) and RMF (redistribution matrix functions) into account ${ }^{2}$, where the RMF were calculated by Monte Carlo simulations (Sturner et al. 2003). This convolution method has been successfully tested using the data obtained from the Crab Nebula observations during revolution 1387 and the results obtained by Jourdain \& Roques (2009). The model convolutions were performed with IRFs and RMFs version 7.0 and the theoretical models used in this work are described in Appendix A and Table A.1. As an example, Fig. 2 presents the DDT1p4 spectrum convolved with the spectral responses of SPI during revolutions 1380-1386, as well as the influence of the off-diagonal terms.

2 For more details on the method, see Compact Source Analysis document: http://www.isdc.unige.ch/integral/download/ osa/doc/10.1/spi_compact_source_analysis.pdf 


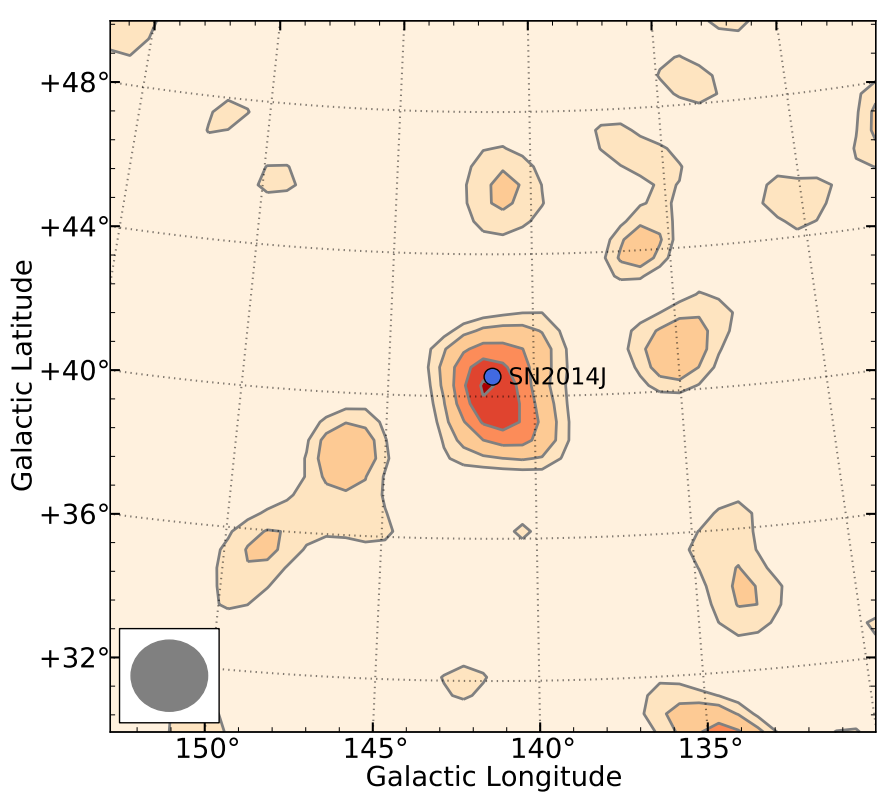

Fig. 3. Gamma-ray signature of SN2014J in the SPI data. The panel displays the statistics map for single detector events (SE) obtained by SPI during the entire early period of observation (days $\sim 16-35$ days after the explosion) over the energy band $145-165 \mathrm{keV}$. The maximum likelihood ratio of the contour lines is $0,2.5,5,10,15$, and 20 . This statistics map has been obtained by fitting a point source on top of the background and the background alone for each position using pixels of 0.5 degrees. The excess in the $\mathrm{SN} 2014 \mathrm{~J}$ position is $5 \sigma$.

The analysis of the data obtained by SPI during this first observation period has revealed an emission excess in the 70-190 and $650-1300 \mathrm{keV}$ bands at the position of SN2014J that was not present in the observations performed by INTEGRAL before the explosion. Figure 3 displays this emission excess in the energy band of $145-165 \mathrm{keV}$, where the $158 \mathrm{keV}{ }^{56} \mathrm{Ni}$ gammaray line is expected to lie. The significance of this excess, $5 \sigma$, is computed by subtracting the log of the maximum likelihood values obtained by fitting both the background alone and the background plus source. The figure also shows that the maximum of the emission coincides with the position of SN2014J, $l=140.5^{\circ}, b=42.5^{\circ}$, and it is clearly isolated from the neighbouring sources as seen by SPI. This localization represents an improvement over the offset of $\sim 2^{\circ}$ present in the previous values reported by Diehl et al. (2014).

In the low-energy region, a broad and completely unexpected redshifted feature associated to the $158 \mathrm{keV}{ }^{56} \mathrm{Ni}$ gamma-ray line has been found in the SPI data (see, however, Diehl et al. 2014, for a different analysis). Figure 4 displays the spectrum obtained during orbits 1380-1386 (16.5-35.2 days after the explosion) by SPI in the $120-190 \mathrm{keV}$ band using two independent procedures and the spectrum predicted by different theoretical models (see Appendix A) after being convolved with the SPI response. All the classical, spherically symmetric models predict a blueshifted line at this epoch, and the continuum, which depends on the adopted model, is in the range of $1.5 \times 10^{-6}$ and $5 \times 10^{-6} \mathrm{ph} \mathrm{cm}^{-2} \mathrm{~s}^{-1} \mathrm{keV}^{-1}$. Figure 4 also shows the concordance between the two independent analyses of data that were performed in the region where the $158 \mathrm{keV}{ }^{56} \mathrm{Ni}$ line should be placed.

Figure 5 presents the spectrum from $20 \mathrm{keV}$ to $1370 \mathrm{keV}$ with a binning of $50 \mathrm{keV}$, where a flux excess in the $720-870 \mathrm{keV}$ energy band can also be seen. The significance

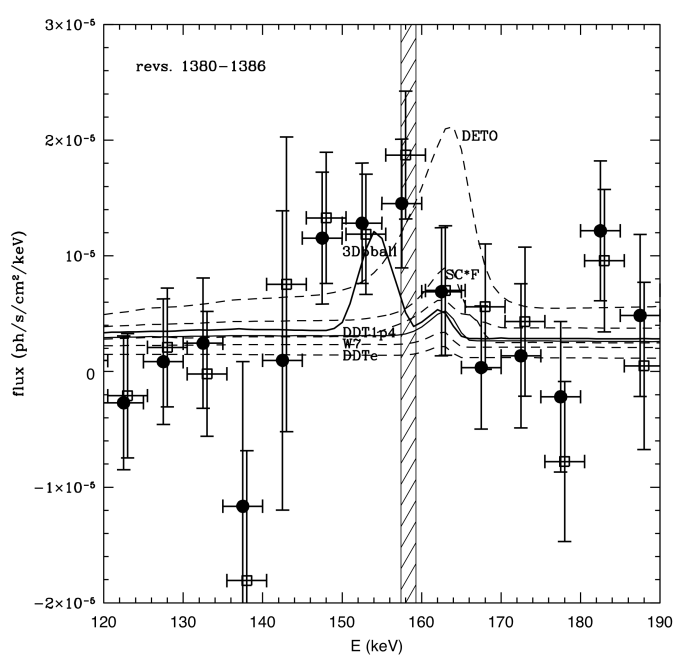

Fig. 4. Spectrum of SN2014J obtained by SPI during revolutions 13801386 in bins of $5 \mathrm{keV}$ in the $120-190 \mathrm{keV}$ band. Filled circles were obtained with the procedures described in Isern et al. (2013) and empty squares as in Churazov et al. (2014b). The last ones have been shifted $0.5 \mathrm{keV}$ for clarity. The lines represent the signal that is expected from a subset of theoretical models listed in Table A.1 (SC*F means SC1F and $\mathrm{SC} 3 \mathrm{~F}$ ) after convolving with the SPI response. The shaded region is centred on the nominal energy of the ${ }^{56} \mathrm{Ni}$ line, $158.4 \mathrm{keV}$, and its width is equal to the energy resolution of SPI at this energy.

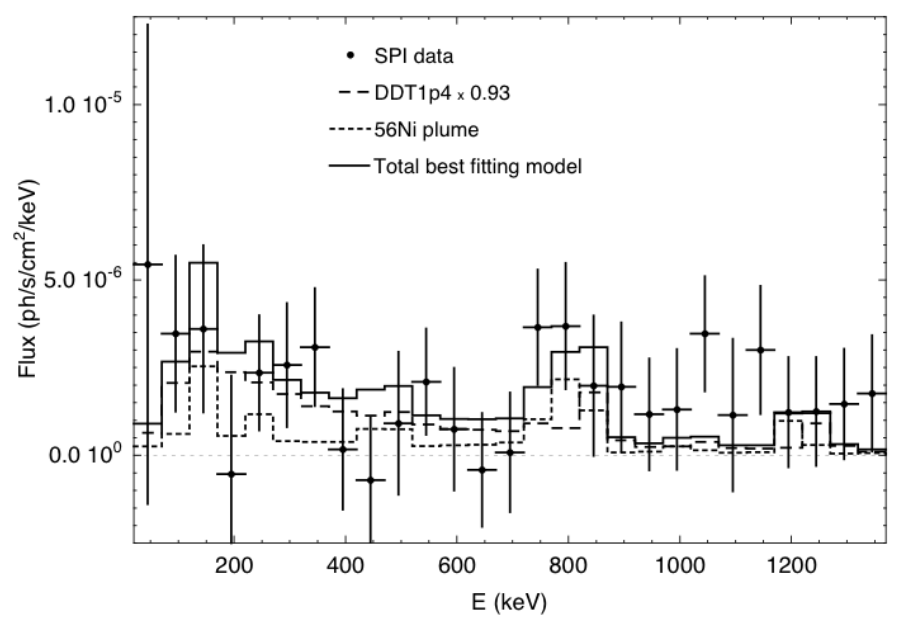

Fig. 5. Gamma-ray spectrum during revolutions 1380-1386 (16.5-35.2 days after the explosion). Bins are $50 \mathrm{keV}$ wide. The continuous line represents the best fit obtained scaling the model DDT1p4 by a factor $0.93\left(0.605 M_{\odot}\right.$ of $\left.{ }^{56} \mathrm{Ni}\right)$ - long dashed line - and adding a ${ }^{56} \mathrm{Ni}$ plume of $0.077 M_{\odot}-$ short dashed line.

of this excess is $\sim 2.8 \sigma$ and can be attributed to the contribution of the ${ }^{56} \mathrm{Ni}$ and ${ }^{56} \mathrm{Co}$ decays. Unfortunately, the blending of the $812 \mathrm{keV}{ }^{56} \mathrm{Ni}$ and $847 \mathrm{keV}^{56} \mathrm{Co}$ lines, caused by the Doppler broadening (Gómez-Gomar et al. 1998), together with the relative weakness of the fluxes, prevents any spectroscopic analysis of these individual gamma-ray lines. It is also interesting to notice the presence of a feature with a $2.6 \sigma$ significance at $\sim 730 \mathrm{keV}$, the position that would correspond to the $750 \mathrm{keV}^{56} \mathrm{Ni}$ line redshifted by the same amount as the $158 \mathrm{keV}$ line (see Fig. 6). The Gaussian fit of this feature gives a flux of $(1.5 \pm 0.7) \times 10^{-4} \mathrm{ph} \mathrm{s}^{-1} \mathrm{~cm}^{-2}(2.1 \sigma)$, a centroid placed at $733.4 \pm 3.8 \mathrm{keV}$, and a FWHM of $16.9 \pm 9.0 \mathrm{keV}$.

If it is assumed that the redshifted feature associated to the $158 \mathrm{keV}$ line is due to ${ }^{56} \mathrm{Ni}$, the other gamma-ray lines emitted 


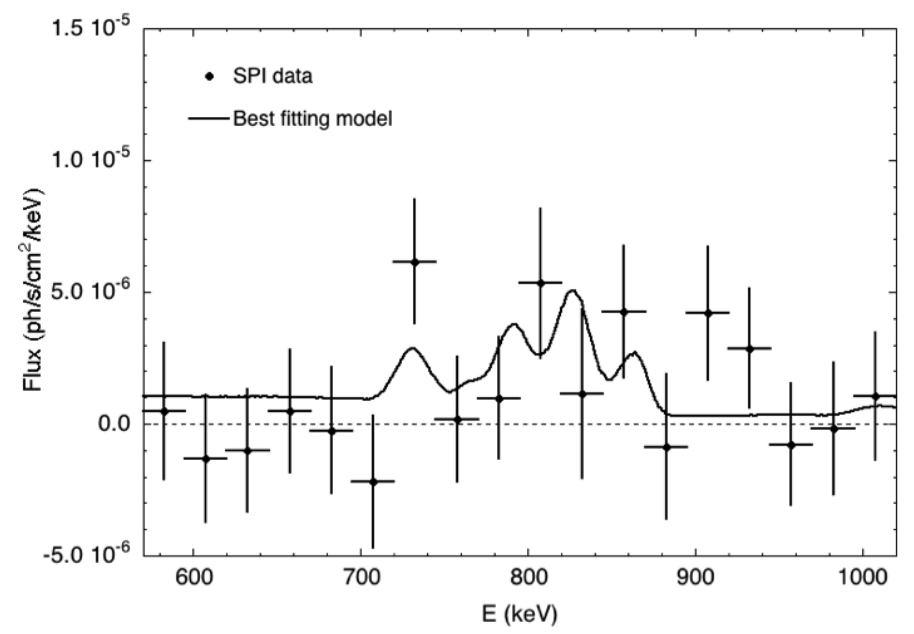

Fig. 6. Gamma-ray spectrum during revolutions 1380-1386 (16.5-35.2 days after the explosion) but with bins of $25 \mathrm{keV}$ size. As in Fig. 5, the continuous line represents the best fitting model convolved with the SPI response.

by this isotope should also be redshifted and their widths and fluxes should be in agreement with those of the $158 \mathrm{keV}$ line taking their branching ratio into account (see below). Therefore, including measured bins of the high energy lines in the spectral analysis provides an additional constraint on the analysis of the ${ }^{56} \mathrm{Ni}$ emission. Consequently, the flux, the width, and the redshift of the $158 \mathrm{keV}$ line were fitted to the data by linking these three parameters to the respective fluxes, broadening, and redshifts of the $750 \mathrm{keV}$ and $812 \mathrm{keV}$ lines with their corresponding branching ratio ( 0.50 and 0.86 , respectively). Under these conditions, the best fit with a Gaussian that links this feature with the redshifted 750 and $812 \mathrm{keV}^{56} \mathrm{Ni}$ lines gives a flux of $(1.6 \pm 0.4) \times 10^{-4} \mathrm{~cm}^{-2} \mathrm{~s}^{-1}$, centred at $155.2_{-1.1}^{+1.3} \mathrm{keV}$ with a FHWM 5.2 $2_{-2.2}^{+3.4} \mathrm{keV}$. These values were obtained from the analysis of the $2 \mathrm{keV}$ bin spectrum (615 bins) between $120 \mathrm{keV}$ and $1350 \mathrm{keV}$. Considering the three free parameters, energy shift, broadening, and flux in the $158 \mathrm{keV}$ line, this gives $\chi^{2}=564.76$ and a reduced $\chi^{2}=0.923$ for a d.o.f. 612. The null hypothesis yields $\chi^{2}=592.25$ : i.e. the $\Delta \chi^{2} \sim 27.5$.

These results contrast with those found by Diehl et al. (2014), who observed two very narrow lines placed at the nominal values of the 158 and $812 \mathrm{keV}^{56} \mathrm{Ni}$ features, so very near to the aforementioned instrumental lines. Fortunately, the $158 \mathrm{keV}$ feature found in this work is broad and is shifted to $\sim 155 \mathrm{keV}$, where there are no such background lines.

The evolution of the spectrum during this early phase of observation could also provide some hints to the nature of the explosion. With such a purpose, data in the 120-190 keV band were grouped into bins corresponding to revolutions 1380-81, 1382-83 and 1384-85. These time intervals were chosen as a compromise between an optimal signal-to-noise ratio and the possibility of solving the light curve in time. Figure 7 displays the Gaussian fits obtained in this way. The flux measured during revolutions $1380-1381$ is $(2.23 \pm 0.80) \times 10^{-4} \mathrm{ph} \mathrm{cm}^{-2} \mathrm{~s}^{-1}$, centred at $152.6 \pm 2.8 \mathrm{keV}$ with a significance of $2.8 \sigma$. In the two other bins, the signal-to-noise ratio is too poor to perform any definite comparison of the evolution of the lines, and only upper limits $\left(2 \sigma\right.$ level) can be provided: $<1.72 \times 10^{-4}$, and $<1.53 \times 10^{-4} \mathrm{ph} \mathrm{cm}^{-2} \mathrm{~s}^{-1}$. In any case, these values are an overestimation of the flux since the intrinsic continuum of SN2014J, detected during the late observations (Churazov et al. 2015),

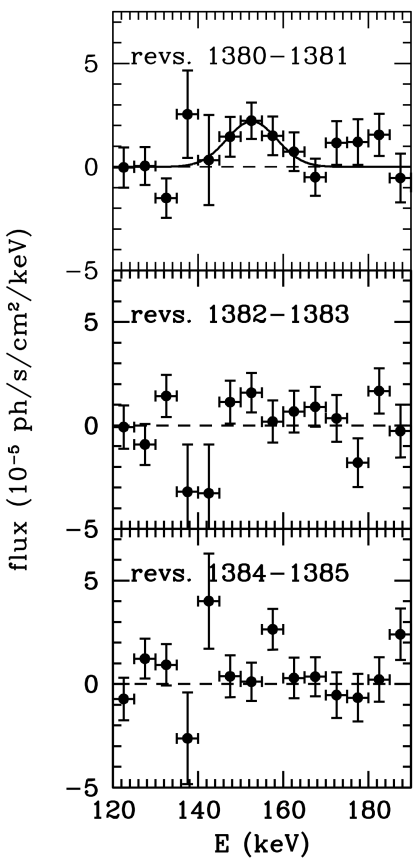

Fig. 7. Spectral evolution of SN2014J obtained by SPI in the $120-190 \mathrm{keV}$ band during revolutions $1380-81,1382-83$, and 1384-85 or 16.5-22.2, 22.6-28.2, and 28.6-35.2 days after the explosion (top to down), respectively, extracted in bins of $5 \mathrm{keV}$.

and the complete spectral response of SPI were not taken into account.

If the intrinsic continuum of SN2014J is considered and the complete response of SPI is adopted, the fluxes in the Gaussian fits of the spectra presented in Fig. 7 become $(1.59 \pm 0.57) \times 10^{-4}$, $<1.42 \times 10^{-4}$, and $<1.56 \times 10^{-4}$ photons $\mathrm{cm}^{-2} \mathrm{~s}^{-1}$ after removing the continuum underneath the line produced by the off-diagonal terms of the DDT1p4 model. In this case, the most significant gamma-ray line signature from the ${ }^{56} \mathrm{Ni}$ occurs during revolutions $1380-1381$ with a centroid at $154.5 \pm 0.64 \mathrm{keV}$ and a width of $3.7 \pm 1.5 \mathrm{keV}$.

\subsection{IBIS/ISGRI data}

As in the case of SPI, the data obtained by IBIS/ISGRI were analysed independently with the method described in Isern et al. (2013), which takes the response of the instrument into account, but using the OSA-10 instead of OSA-9 since it noticeably improves the reconstruction of the photon energy, and with the method described in Churazov et al. (2014b), where the flux is obtained by normalizing to the values of the Crab in the same energy band. Usually, this normalization procedure is sufficient if the energy band being analysed is broad enough, but this is only strictly valid if both spectra, Crab and supernova, are similar, which is not the case. For this reason, the procedure adopted here is to compare the observations with the theoretical models convolved with the ISGRI spectral response. The energy resolution at $155 \mathrm{keV}$ was $F W H M \sim 14 \mathrm{keV}$, but due to the detector degradation in orbit, the resolution is now closer to $20 \%$ (Caballero et al. 2012). To show how the evolution of the count rate depends on the spectrum of the adopted models and how they evolve with time, Fig. 8 displays the count rate that is obtained from three of the models used here after convolving with the response of the instrument, and the count rate obtained just 


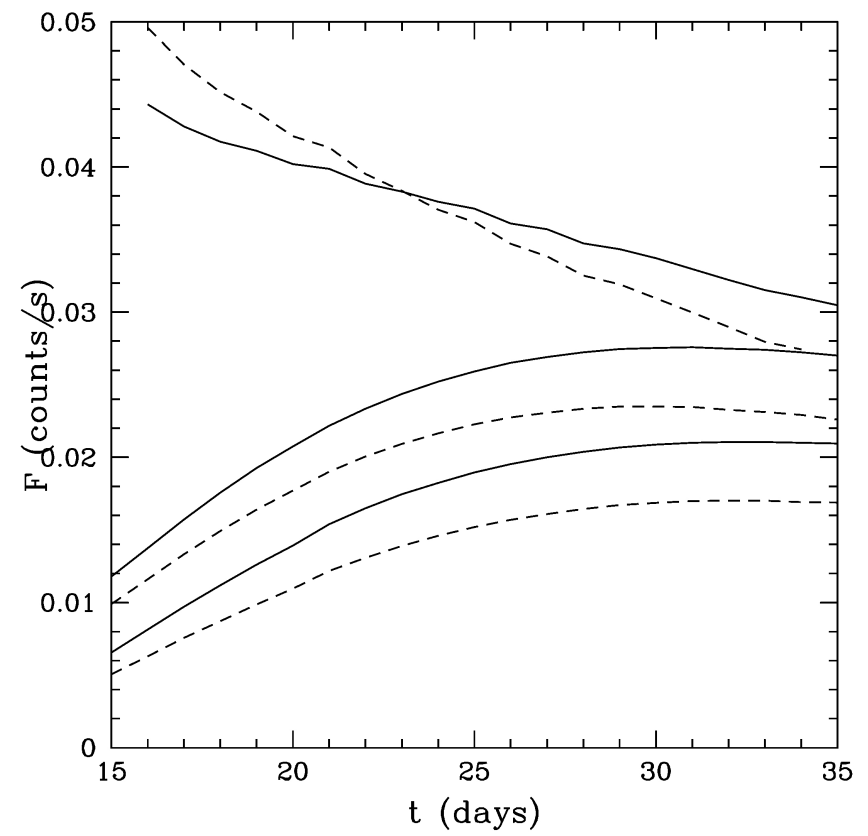

Fig. 8. IBIS/ISGRI light curve in response to different spectral models. Dashed lines represent the temporal evolution of the $144.5-168 \mathrm{keV}$ band of the 3Dbball, DDT1p4, and W7 models (from top to bottom) multiplied by the effective area in this band $\left(370 \mathrm{~cm}^{2}\right)$. Continuous lines represent their convolution with the ISGRI response.

multiplying the theoretical fluxes by the effective area in the energy band under consideration.

When taking the ISGRI spectral response into account, the signal expected from most models is maximum over the $68-190 \mathrm{keV}$ range, and the observations performed by IBIS/ISGRI during the same period of time as SPI reveal an emission excess at the position of SN2014J in the energy band 67.5-189 keV. Figure 9, upper panel, clearly shows that this emission excess cannot be confused with the neighbouring sources. However, if the analysis is restricted to the 144.4-168 keV band, the significance of the signal decreases to about 2 sigma, as expected from the ISGRI spectral response (see Fig. 9, lower panel). In the $25-70 \mathrm{keV}$ band nothing is visible at the position of SN2014J.

Figure 10 displays the response of ISGRI to an incoming gamma-ray flux that has the same spectrum as one of the models used in this work, the 3Dbball model. As can be seen, the photons belonging to the $158 \mathrm{keV}{ }^{56} \mathrm{Ni}$ lines are redistributed over a spectral band that is larger than expected. As a consequence, the flux of the line weakens when a narrow spectral window is taken.

Given the strong redistribution of photons, only the broad band of $67.5-189 \mathrm{keV}$ will be considered. Table 2 and Fig. 11 display the temporal evolution of the count rate measured by IBIS/ISGRI in this band, which is dominated by the $158 \mathrm{keV}$ ${ }^{56} \mathrm{Ni}$ emission. As in the case of SPI, bins are roughly six days wide and correspond to revolutions 1380-81, 1382-83, and 138485 . The behaviour, similar to that obtained by SPI but with a better significance, suggests a decline in the count rate that is compatible with the non-absorbed emission of ${ }^{56} \mathrm{Ni}$, followed by an upturn at the end of the observation period that could be the consequence of the exposure of new radioactive layers. Unfortunately, the poor $\mathrm{S} / \mathrm{N}$ of the central bin $(1.9 \sigma)$ prevents any solid conclusion about this point, and an approximately constant or gently decaying behaviour cannot be excluded.
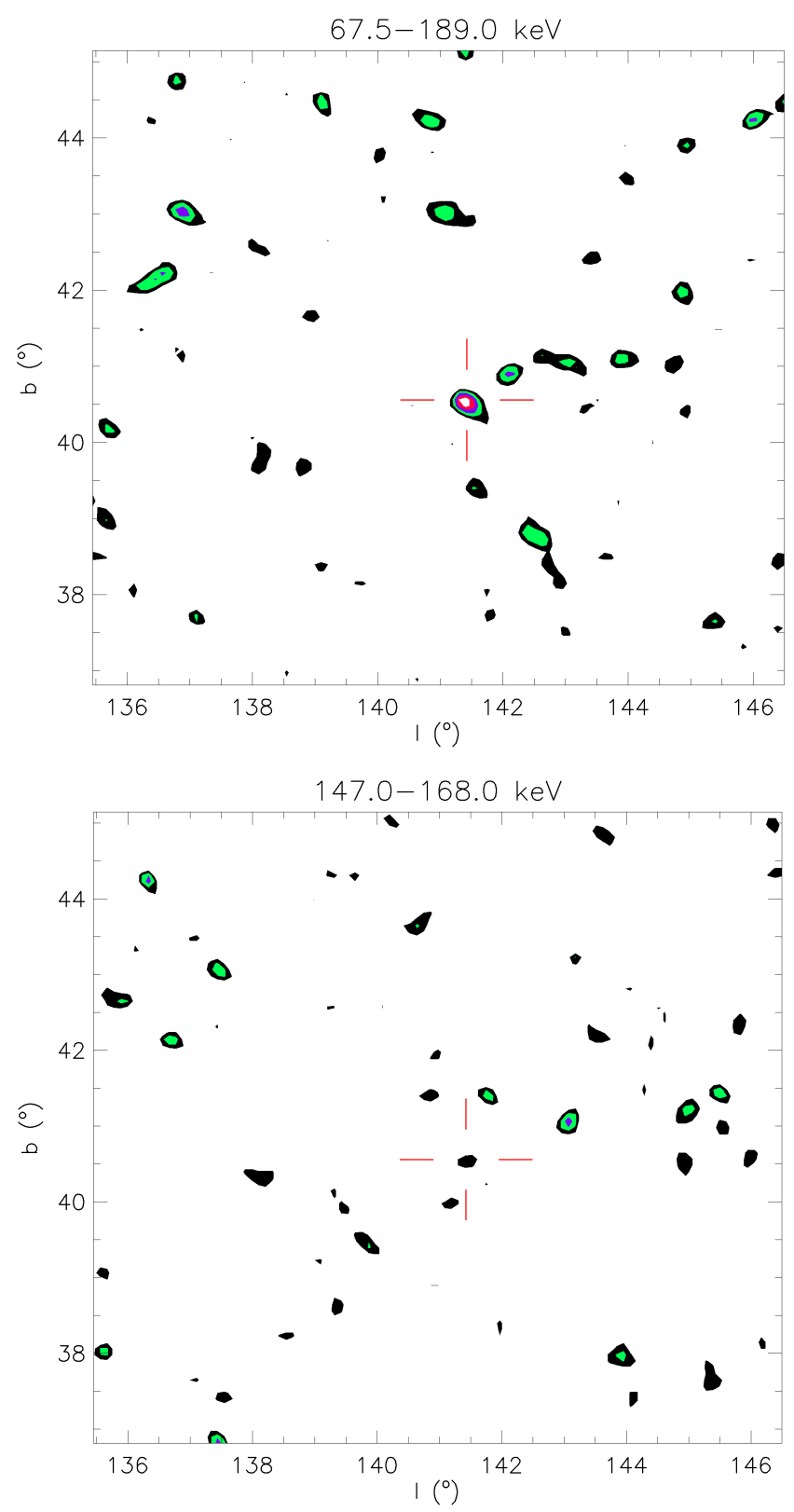

Fig. 9. Gamma-ray signature in the IBIS/ISGRI data. The figure displays the IBIS/ISGRI significance contour map in the $67.5-189 \mathrm{keV}$ band (upper panel) and in the 147-168 keV band (lower panel) for the entire early period (days 16-35 after the explosion). The contours start at the $2 \sigma$ level and are separated by $0.5 \sigma$. The average flux in the upper panel at the position of SN2014J represents a $5.4 \sigma$ excess after normalization on the standard deviation observed in this map.

\section{Results and discussion}

There are several spherically symmetric SNIa models (see Appendix A) with the bulk of radioactive elements buried in the central layers of the expanding debris that are able to reproduce, with the appropriate parameters, the ${ }^{56} \mathrm{Co}$ features observed at late times, 55-100 days after the explosion in SN2014J (Churazov et al. 2014b, 2015). After being convolved with the SPI response, as in the case of DDT1p4 presented in Fig. 5, these models can be compared with the observed spectrum taken during revolutions 1380 to 1386 in the range $120-1350 \mathrm{keV}$. The degrees of freedom are 246, and Table 3 presents the resulting $\chi^{2}$ values. 


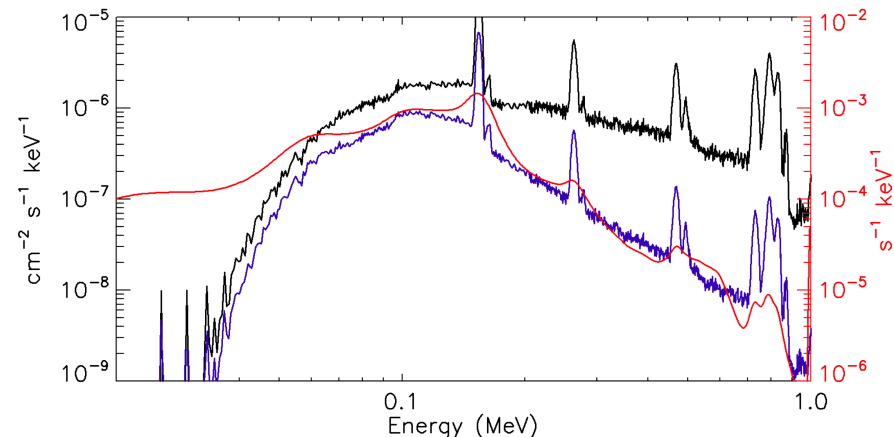

Fig. 10. Response of IBIS/ISGRI to an incoming gamma signal that has a characteristic supernova spectrum like the one provided by the 3Dbball model (black line). The blue line is obtained by multiplying this spectrum by the ARF (auxiliary response files) that represent the effective area, and finally the red line represents the values obtained by convolving this last result with the RMF (redistribution matrix function).

Table 2. Temporal evolution of the $158 \mathrm{keV}{ }^{56} \mathrm{Ni}$ according to IBIS/ISGRI.

\begin{tabular}{cccc}
\hline \hline Revolutions & Days & Counts/s & $S / N$ \\
\hline $1380-1381$ & $22.20-16.50$ & $0.149 \pm 0.039$ & 3.8 \\
$1382-1383$ & $28.20-22.60$ & $0.078 \pm 0.041$ & 1.9 \\
$1384-1385$ & $34.20-28.60$ & $0.143 \pm 0.037$ & 3.8 \\
\hline
\end{tabular}

Table 3. Comparison between models and the spectra measured by SPI (d.o.f. $=246$ ). $H_{0}$ represents the null hypothesis.

\begin{tabular}{cc}
\hline \hline Model & $\chi^{2}$ \\
\hline$H_{0}$ & 250.1 \\
DETO & 269.2 \\
W7 & 229.5 \\
DDTe & 237.0 \\
SC1F & 226.4 \\
SC3F & 227.9 \\
\hline DDT1p4 & 227.2 \\
3Dbball & 220.8 \\
\hline
\end{tabular}

The DDT1p4 model explains the optical light curve (see section 2) and the gamma-ray emission at late epoch (e.g. Churazov et al. 2014). If, in order to make a crude comparison and to use the same criteria as in Churazov et al. (2015), we adopt this model as a reference, we see that DETO differs by $\sim 6 \sigma$ and DDTe by $\sim 3 \sigma$ level, while the remaining ones are nearly as good as the DDT1p4 model $(<1.5 \sigma)$. However, despite the reasonable agreement with the observed values of these remaining models, they are neither able to reproduce the intensity and the redshifted position of the $158 \mathrm{keV}{ }^{56} \mathrm{Ni}$ line observed by SPI (Fig. 4) nor the excess of emission in the bin corresponding to the 1380-81 orbits found in the IBIS/ISGRI data (Fig. 11) and, in a less compelling form, by SPI (Fig. 7). Only DETO seems to fulfil these last requirements, but it synthesizes a total amount of ${ }^{56} \mathrm{Ni}$ that is too large to account for the late emission of ${ }^{56} \mathrm{Co}$. It also predicts the presence of important amounts of this isotope in the outer layers that contradicts the optical observations during the maximum of the light curve. Therefore, it seems natural to propose models with small amounts of radioactive material in the outer layers of the supernova debris (Burrows \& The 1990; Gómez-Gomar et al. 1998) that are undetectable at the other wavelengths.

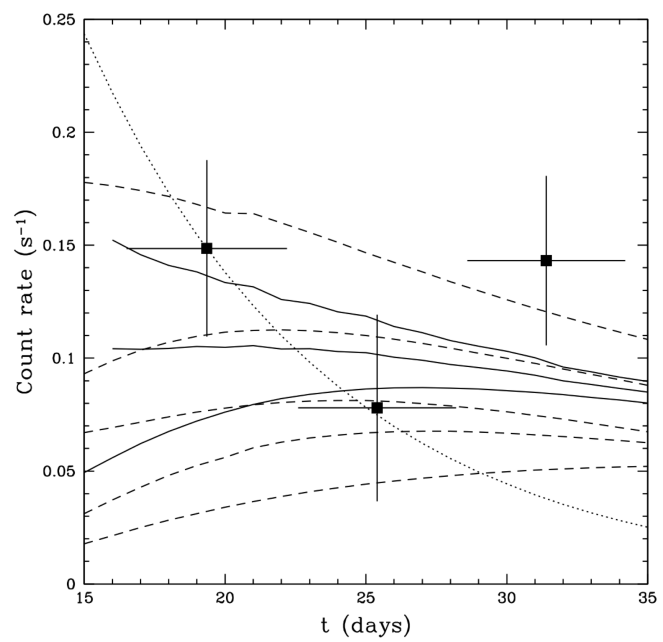

Fig. 11. Evolution of the 67.5-189 keV band during revolutions 1380 $81,1382-83$, and $1384-86$ as obtained by IBIS/ISGRI using the method described in Isern et al. (2013). The dotted line represents the flux that would be provided by the free disintegration of ${ }^{56} \mathrm{Ni}$. Dashed lines represent the light curves obtained by convolving models DETO, SC3F, $\mathrm{SC} 1 \mathrm{~F}, \mathrm{~W} 7$, and DDTe (from top to down) with the instrument response. Solid lines represent, from top to down, the 3Dbball with plumes of 0.08 and $0.04 M_{\odot}$ of ${ }^{56} \mathrm{Ni}$, and the DDT1p4 models after convolution. The properties of these models are displayed in Appendix A.1.

The average $3.2 \mathrm{keV}$ redshift, $\sim 2 \%$ of the nominal $158 \mathrm{keV}$ energy, indicates that the material is receding from the observer with a mean velocity $v \approx 6000 \mathrm{~km} \mathrm{~s}^{-1}$ and is placed in the far hemisphere, while the measured average width, $4.9 \mathrm{keV}$, implies a maximum deviation of the component of the velocity along the line of sight of $\Delta v \approx 10000 \mathrm{~km} \mathrm{~s}^{-1}$. Nothing can be said about the velocity in the plane normal to the line of sight except that, in order to not be caught by the outer layers of the supernova, it must have a velocity of $\sim 30000 \mathrm{~km} \mathrm{~s}^{-1}$. This possibility could be supported by the rapid rise of the optical light curve at early times (Zheng et al. 2014; Goobar et al. 2014) and by the microvariability found 15-18 days after the maximum in the Blight curve (Bonanos \& Boumis 2016) in SN2014J, by the chemical inhomogeneities found in Kepler (Reynolds et al. 2007) and Tycho (Vancura et al. 1995; Warren et al. 2005) remnants and by the properties of the high velocity features detected in the early optical spectra, about ten days after the explosion, of many SNIa (Tanaka et al. 2006).

This radioactive material should be almost transparent to gamma-rays at the time of the INTEGRAL observation near the maximum of the optical light curve, otherwise it would have been detected in the optical. This condition is also necessary to account for the dip suggested by IBIS and SPI data about 25 days after the explosion (Figs. 11 and 7). Furthermore, the not detecting blueshifted Ni-Co features in the optical and in the infrared at this epoch indicates that it was not placed between the observer and the supernova. An additional argument in favour of the transparency hypothesis is that an opaque plume would demand a higher ${ }^{56} \mathrm{Ni}$ mass to obtain a similar flux, and this would introduce a redshifted component in the late ${ }^{56} \mathrm{Co}$ emission that is not observed (Churazov et al. 2014b, 2015).

The first obvious geometry choice to be considered is a spherical blob that broke away from the bulk of the supernova ejecta. Such a configuration, however, does not guarantee the transparency of the blob at the moment of the observation. For instance, Fig. 12, upper panel, displays a blob with a mass 

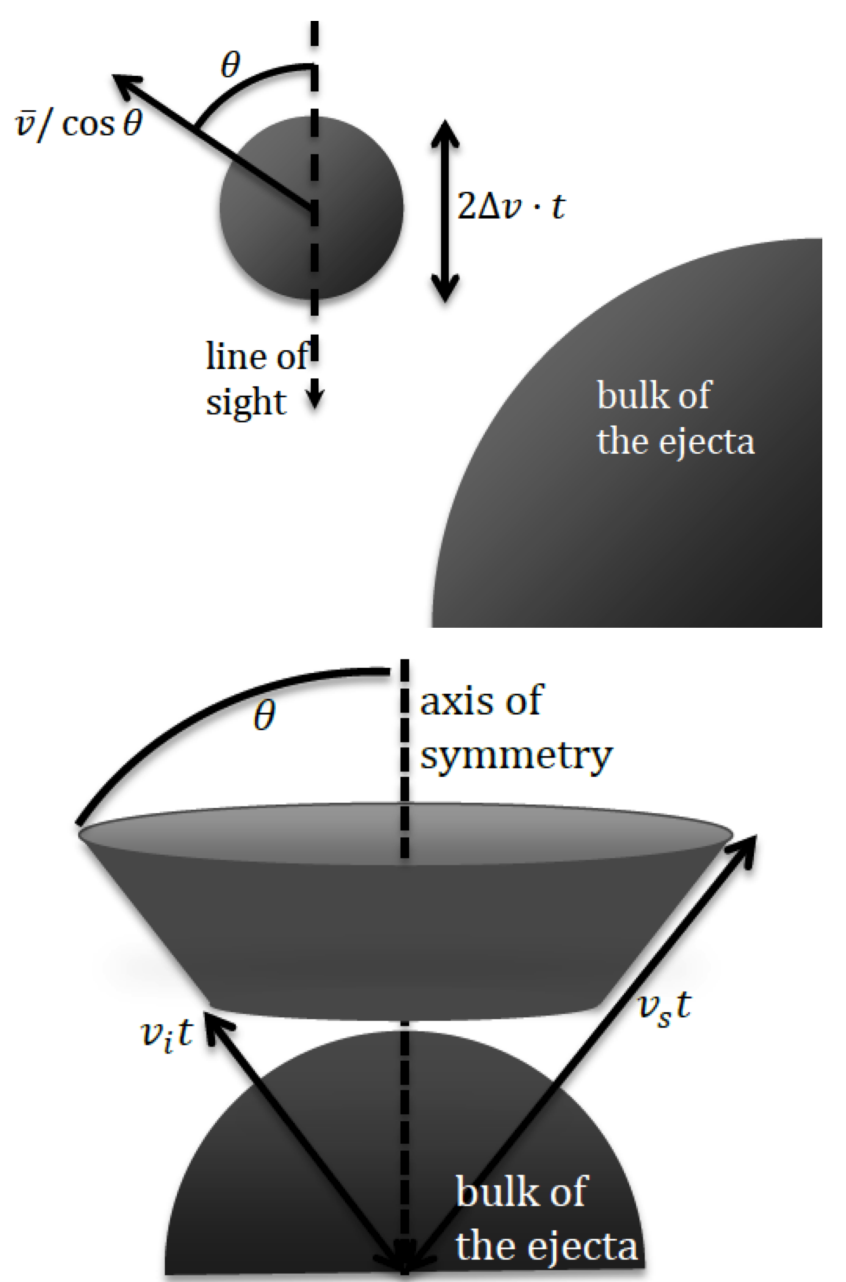

Fig. 12. Possible geometries of the outer radioactive layers. The upper panel represents a blob with a mass $M \sim 0.05 M_{\odot}$ that detached from the main body during the explosion and moved with a velocity compatible with the observed redshift. The lower panel represents a plume with the shape of a truncated conical ring that has a semiaperture angle $\theta$ and angular thickness $\Delta \theta$.

$M \sim 0.05 M_{\odot}$, expelled by the main body of SN2014J with a velocity of $\sim 30000 \mathrm{~km} \mathrm{~s}^{-1}$ and increasing its radius with a velocity of $\Delta v \sim 10000 \mathrm{~km} \mathrm{~s}^{-1}$, the internal velocity dispersion. At day 18 after the explosion, the radius of the ball should be $\Delta v \times t=1.5 \times 10^{15} \mathrm{~cm}$ and the optical depth $\tau=\kappa \Sigma \approx 3$, where $\Sigma \sim M / \pi R^{2} \approx 14 \mathrm{~g} \mathrm{~cm}^{-2}$. Assuming constant density, and $\kappa \approx 0.2 \mathrm{~cm}^{2} \mathrm{~g}^{-1}$ for the $158 \mathrm{keV}$ line, the blob would be opaque to the gamma-ray radiation at the moment of the observation and should be detectable in the optical.

A more favourable geometry is to distribute the radioactive matter in a ring with a truncated conical shape as depicted in the lower panel of Fig. 12. We call this model 3Dbball. This conical structure has the same mass as before, and in order to be compatible with the observations of SN2014J, a semi-aperture angle $\theta \approx 78^{\circ}$ and an angular thickness $\Delta \theta \approx 12^{\circ}$ were adopted as an example, although other possibilities do exist. Similarly, the expansion velocities were set to be $v_{\mathrm{i}} \sim 25000 \mathrm{~km} \mathrm{~s}^{-1}$ and $v_{\mathrm{s}} \sim 35000 \mathrm{~km} \mathrm{~s}^{-1}$ in order to fulfil the requirements. In this case, the column density would be $\Sigma=M / 4 \pi\left(R_{\mathrm{S}}-R_{\mathrm{i}}\right)^{2} \sin \theta \sim$ $2.4 \mathrm{~g} \mathrm{~cm}^{-2}$, where $R_{\mathrm{s}, \mathrm{i}}=v_{\mathrm{s}, \mathrm{i}} t$, and the optical depth would be 0.48 , which would make the material of the plume optically thin to the $158 \mathrm{keV}$ photons.

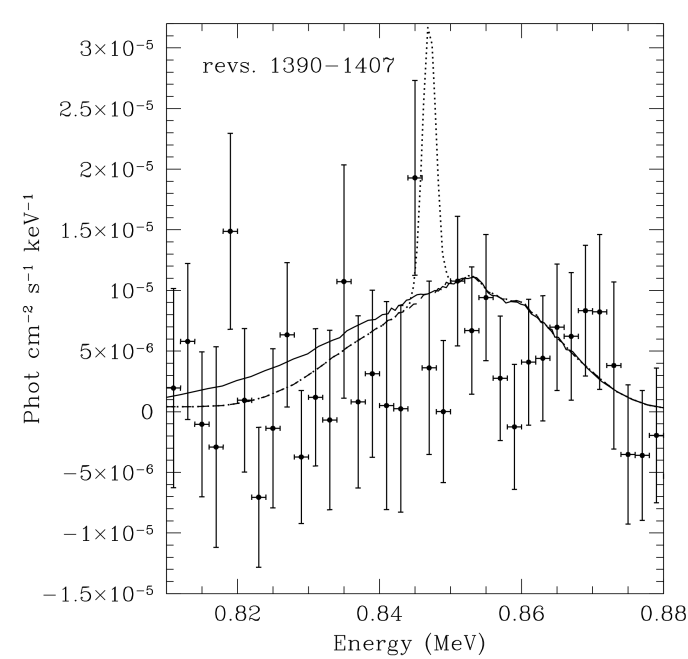

Fig. 13. High resolution gamma-ray spectrum 50-100 days after the explosion around the $847 \mathrm{keV}^{56} \mathrm{Co}$ line. Bins are $2 \mathrm{keV}$ wide. The dashed line represents the DDT1p4 model. The solid line represents the emission of the 3Dbball model (the DDT1p4 plus a plume of $0.07 M_{\odot}$ of ${ }^{56} \mathrm{Ni}$ out the equatorial plane). The dotted line represents the DDT1p4 model plus a plume of $0.06 M_{\odot}$ of ${ }^{56} \mathrm{Ni}$ confined within the equatorial plane. All of them have been computed seventy five days after the explosion.

Assuming the complete transparency to gamma-rays, the total mass of ${ }^{56} \mathrm{Ni}$ can be estimated to be

$$
\begin{aligned}
m_{0}= & 4 \pi D^{2}\left(\frac{56}{N_{\mathrm{A}}}\right)\left[\frac{\left\langle F_{158}\right\rangle\left(t_{\mathrm{i}}-t_{0}\right)}{Y_{158}}\right] \\
& \times\left[\exp \left(-t_{0} / \tau_{\mathrm{Ni}}\right)-\exp \left(-t_{\mathrm{i}} / \tau_{\mathrm{Ni}}\right)\right]^{-1}
\end{aligned}
$$

where $N_{\mathrm{A}}$ is the Avogadro's number, $D$ the distance, $Y_{158}$ the branching ratio, $t_{0}$ and $t_{\mathrm{i}}$ the beginning and the end times of the observation, $\tau_{\mathrm{Ni}}$ the characteristic decay time and $\left\langle F_{158}\right\rangle$ the average flux in this time interval. The flux measured by SPI during revolutions $1380-1381$ indicates that the mass in the plume should be $\sim 0.07 M_{\odot}$ while the mass needed to account for the flux measured by ISGRI, when the deconvolution method is used, is $\sim 0.08 M_{\odot}$. These values seem to give support to the hypothesis that ${ }^{56} \mathrm{Ni}$ is present in the outer layers. There is also a hint, provided by the increase in the intensity of the line flux during revolutions 1384-1385, of the emergence of a new, deeper, plume or of the exposure of the internal radioactive core, but the lack of enough significance of the signal prevents any firm conclusion.

The presence of a plume able to produce the broad redshifted ${ }^{56} \mathrm{Ni}$ lines claimed here is compatible with the observed properties of the ${ }^{56}$ Co lines (Churazov et al. 2014b, 2015) at late times. Figure 13 shows at high resolution $(2 \mathrm{keV})$ the flux averaged over days 50 to 100 after the explosion. At this epoch the debris are almost transparent to gamma rays and the presence of the plume has a negligible effect on the spectrum since its contribution is smeared over a large energy interval as a consequence of its broadness. In contrast, if the plume had contained almost all this amount of ${ }^{56} \mathrm{Ni}$ in the equatorial plane and was seen perpendicularly, it would have produced a prominent spike similar to or narrower than the one represented in the figure. Such a spike would not have introduced dramatic effects at low resolution, but at the resolution provided by SPI, it should be detectable. Just as an example, adding a ring of $0.06 M_{\odot}$ of ${ }^{56} \mathrm{Ni}$ in the equatorial plane able to produce a narrow $(F W H M \approx 2.23 \mathrm{keV})$ feature at $812 \mathrm{keV}$ when ${ }^{56} \mathrm{Ni}$ was detected should produce, 75 days after the explosion, a flux at the nominal energy of the $847 \mathrm{keV}$ 
${ }^{56} \mathrm{Co}$ line $\sim 4.4 \sigma$ larger than the observed value, as can be seen in Fig. 13, which can be interpreted as a rejection of such a model with a probability greater than $99.9 \%$. The maximum mass of ${ }^{56} \mathrm{Ni}$ that could be confined within the equatorial plane is estimated to be $\sim 0.02 M_{\odot}(2 \sigma$ level $)$.

The spectral model adopted to extract the line fluxes and characteristics from these early observations is the best-fitting 3Dbball model. As stated in the Appendix, this model is a combination of the DDT1p4 model, which reproduces the ${ }^{56} \mathrm{Co}$ gamma-ray lines observed in Late Observations of SN2014J (Churazov et al. 2014b) and the optical light curve, and a ${ }^{56} \mathrm{Ni}$ plume that accounts for the observed redshifted line. The best fit to the SPI data (120-1350 keV band) is obtained with a mass of ${ }^{56} \mathrm{Ni}$ of $0.077 \pm 0.040 M_{\odot}$ in the plume and $0.60 \pm 0.29 M_{\odot}$ in the central body (Fig. 5). These best fit values were obtained with a $\chi^{2}=210.72$ for a d.o.f. of 244 (246 spectral bins and 2 free parameters: the mass of ${ }^{56} \mathrm{Ni}$ in the plume and in the central body). This last value for the central body agrees with the mass derived from the optical light curve measurements (Sect. 2) and with the mass obtained with the analysis of the gamma-ray emission at the late epoch (Churazov et al. 2014b).

Finally, it is worth mentioning that if this 3Dbball model is adopted as a reference, DETO and DDTe models can be rejected at the $\sim 7 \sigma$ and $\sim 4 \sigma$ levels, respectively, while the remaining ones differ by $<3 \sigma$ and cannot be formally rejected (Table 3). Also, Fig. 6 shows that the flux excess found at $\sim 730 \mathrm{keV}$, which was attributed to the redshifted $750 \mathrm{keV}{ }^{56} \mathrm{Ni}$ line in Sect. 2 with a significance of $2.1 \sigma$ ), represents a flux of $(1.2 \pm 0.7) \times 10^{-4} \mathrm{ph} \mathrm{s}^{-1} \mathrm{~cm}^{-2}(\sim 1.7 \sigma)$, when the continuum underneath the line is included in the fit. Although the significance of the $730 \mathrm{keV}$ line flux is not enough to claim its detection, this excess reinforces the plausibility the ${ }^{56} \mathrm{Ni}$ plume hypothesis.

\section{Conclusions}

SN2014J has been observed with all the instruments on board INTEGRAL just around the maximum of the optical light curve for a period of $\sim 10^{6} \mathrm{~s}$. The optical light curve measured with the OMC agrees with the light curves obtained from the ground and can be explained by a delayed detonation model that synthesizes $\sim 0.65 M_{\odot}$ of ${ }^{56} \mathrm{Ni}$ (see Fig. 1).

As previously stressed (Churazov et al. 2015), SN2014J is a weak gamma-ray source, despite its distance, and the results are sensitive to various aspects of the data analysis. The main improvement with respect to previous analysis (Diehl et al. 2014) is the clear detection of the gamma-ray signal by both instruments, SPI and ISGRI, with significances of $5 \sigma$ (see Figs. 3 and 9) during this period of observation at the position of SN2014J, removing the $\sim 2^{\circ}$ offset present in the previous analysis, and confirming the idea that the light curves of SNIa are powered by the decay of ${ }^{56} \mathrm{Ni}$. Surprisingly, we found in the SPI data evidences of a broad, redshifted feature that corresponds to the $158 \mathrm{keV}$ emission of this isotope. Given this energy and broadness, this feature cannot be confused with any residual instrumental line. Furthermore, we also found in the ISGRI data an emission excess during orbits $1380-81$ and $1384-85$ at a $3.8 \sigma$ level (Table 2). These emission excesses are well above the predictions of conventional 1D models (Fig. 11) and are separated by a dip that is compatible with the free decay of ${ }^{56} \mathrm{Ni}$, although the low significance of this dip prevents any definite consideration about the temporal variability. A similar behaviour is suggested by SPI data, an excess of emission followed by a decline, but once more the poor significance prevents any definite conclusion. A possible explanation of this behaviour is that during the SN2014J event, an almost $\gamma$-ray transparent plume made of $\sim 0.08 M_{\odot}$ of ${ }^{56} \mathrm{Ni}$ was ejected with an expansion velocity of $\sim 30000 \mathrm{~km} \mathrm{~s}^{-1}$ and a dispersion velocity of $\sim 10000 \mathrm{~km} \mathrm{~s}^{-1}$, the whole plume receding from the observer with a velocity of $\sim 6000 \mathrm{~km} \mathrm{~s}^{-1}$. The significance of this additional ${ }^{56} \mathrm{Ni}$, which was obtained by fitting the redshifted and broadened $158 \mathrm{keV}, 750 \mathrm{keV}$, and $812 \mathrm{keV}$ lines above the DDT1p4 model with SPI data, is $\sim 3 \sigma$ (see Fig. 4).

Churazov et al. (2014b, 2015) report that the fluxes and spectra of the gamma-rays emitted by ${ }^{56} \mathrm{Co}$ during the maximum of the light curve broadly agree with the predictions of classical spherically symmetric theoretical models of SNIa based on either the deflagration or the delayed detonation paradigms. Figure 13 shows that the introduction of an extra emission caused by the existence of a radioactive plume with the characteristics proposed here predicts a late-time spectrum that is still in accordance with the spectrum obtained during the late epoch (55-100 days after the explosion).

In any case, the significance of the signal prevents any firm conclusion about the behaviour of phenomena changing on a time scale close to the ${ }^{56} \mathrm{Ni}$ decay time. When we take Table 3 into account and adopt a conservative point of view, the data are largely consistent with the standard delayed detonation model (Churazov et al. 2015) without excluding the presence of small amounts of ${ }^{56} \mathrm{Ni}$ at the surface if the lines are broad.

It is evident that if the significance of the redshift and the width of ${ }^{56} \mathrm{Ni}$ lines found in the observations of INTEGRAL was enough, the gamma-ray behaviour would introduce strong constraints on the acceptable models for SN2014J. If confirmed in other supernovae, it could be concluded that conventional models starting with the ignition of the central regions of a $\mathrm{C} / \mathrm{O}$ white dwarf and keeping the radioactive material confined in the innermost layers would not be appropriate to account for the observed properties at this early epoch and that, at least in these cases, additional possibilities should be considered. Sub-Chandrasekhar models - i.e. $\mathrm{C} / \mathrm{O}$ white dwarfs with a mass not necessarily near the critical mass that explode as a consequence of the ignition of a freshly accreted He-envelope (Woosley \& Weaver 1994) - produce ${ }^{56} \mathrm{Ni}$ at the surface and could, in principle, account for the observations if the mass of these layers is low enough (Pakmor et al. 2013; Guillochon et al. 2010; Fink et al. 2010; García-Senz et al. 1999). Three-dimensional models, such as pulsating reverse detonations (PRD; Bravo \& García-Senz 2009; Bravo et al. 2009), gravitationally confined detonations (GCD; Plewa et al. 2004), and collisions of white dwarfs in double-degenerate binaries or multiple systems (Kushnir et al. 2013; Aznar-Siguán et al. 2013) could also provide scenarios with ${ }^{56} \mathrm{Ni}$ present in the outer layers, although in the last case the collision would require the presence of massive white dwarfs to achieve the observed amount of ${ }^{56} \mathrm{Ni}$ (García-Senz et al. 2013). Obtaining similarly extensive INTEGRAL data on additional SNIa would be of the maximum interest not only to ascertain if SN2014J is a representative event, but also to constrain the models for SN2014Jlike events. Nevertheless, given the existence of several SNIa subtypes, only a high sensitivity detector would be able to provide a statistically representative sample of gamma observations. Finally, it is necessary to emphasize that the implications of the reported asymmetrical features on cosmological applications of SNIa still have to be determined.

Acknowledgements. This work was supported by the MINECO-FEDER grants ESP2013-47637-P (JI), AYA2012-39362-C02-01 (AD), AYA2013-40545 (EB), ESP2013-41268-R (MH), AYA2014-59084-P (DGS) and AYA2011-22460 (ID) by the ESF EUROCORES Program EuroGENESIS (MINECO grant EUI200904170), by the grant 2009SGR315 of the Generalitat de Catalunya (JI), and by 
the Ministerium für Bildung und Forschung via the DLR grant 50.OG.9503.0. NER acknowledges the support from the European Union Seventh Framework Programme (FP7/2007-2013) under grant agreement n. 267251 "Astronomy Fellowships in Italy" (AstroFIt). NER is also partially supported by the PRININAF 2014 with the project "Transient Universe: unveiling new types of stellar explosions with PESSTO”. The SPI project has been completed under the responsibility and leadership of the CNES, France. ISGRI was realized by the CEA with the support of CNES. We acknowledge the INTEGRAL Project Scientis Erik Kuulkers (ESA, ESAC) and the ISOC for their scheduling efforts, as well as the INTEGRAL Users Group for their support in the observations.

\section{References}

Arnett, D. 1996, Supernovae and Nucleosynthesis (Princeton University Press) Aznar-Siguán, G., García-Berro, E., Lorén-Aguilar, P., José, J., \& Isern, J. 2013, MNRAS, 434, 2539

Bachetti, M., Harrison, F. A., Walton, D. J., et al. 2014, Nature, 514, 202

Badenes, C., Borkowski, K. J., \& Bravo, E. 2005, ApJ, 624, 198

Bonanos, A. Z., \& Boumis, P. 2016, A\&A, 585, A19

Bravo, E., \& García-Senz, D. 2009, ApJ, 695, 1244

Bravo, E., García-Senz, D., Cabezón, R. M., \& Domínguez, I. 2009, ApJ, 695, 1257

Burrows, A., \& The, L.-S. 1990, ApJ, 360, 626

Caballero, I., Zurita Heras, J. A., Mattana, F., et al. 2012, in Proc. An INTEGRAL view of the high-energy sky (the first 10 years) - 9th INTEGRAL Workshop and celebration of the 10th anniversary of the launch (INTEGRAL 2012), 15-19 October, 142

Churazov, E., Sunyaev, R., Grebenev, S., et al. 2014a, ATel, 5992, 1

Churazov, E., Sunyaev, R., Isern, J., et al. 2014b, Nature, 512, 406

Churazov, E., Sunyaev, R., Isern, J., et al. 2015, ApJ, 812, 62

Clayton, D. D., Colgate, S. A., \& Fishman, G. J. 1969, ApJ, 155, 75

Diehl, R., Siegert, T., Hillebrandt, W., et al. 2014, Science, 345, 1162

Fink, M., Röpke, F. K., Hillebrandt, W., et al. 2010, A\&A, 514, A53

Fossey, J., Cooke, B., Pollack, G., Wilde, M., \& Wright, T. 2014, Central Bureau Electronic Telegrams, 3792, 1

García-Senz, D., Bravo, E., \& Woosley, S. E. 1999, A\&A, 349, 177

García-Senz, D., Cabezón, R. M., Arcones, A., Relaño, A., \& Thielemann, F. K. 2013, MNRAS, 436, 3413

Gómez-Gomar, J., Isern, J., \& Jean, P. 1998, MNRAS, 295, 1

Goobar, A., Johansson, J., Amanullah, R., et al. 2014, ApJ, 784, L12

Guillochon, J., Dan, M., Ramirez-Ruiz, E., \& Rosswog, S. 2010, ApJ, 709, L64
Hillebrandt, W., Kromer, M., Röpke, F. K., \& Ruiter, A. J. 2013, Frontiers of Physics, 8, 116

Höflich, P., Wheeler, J. C., \& Khokhlov, A. 1998, ApJ, 492, 228

Höflich, P., Gerardy, C. L., Fesen, R. A., \& Sakai, S. 2002, ApJ, 568, 791

Isern, J., Bravo, E., \& Hirschmann, A. 2008, New Astron. Rev., 52, 377

Isern, J., Hernanz, M., \& José, J. 2011, in Lect. Notes Phys. 812, eds. R. Diehl, D. H. Hartmann, \& N. Prantzos, (Berlin: Springer-Verlag), 233 Isern, J., Jean, P., Bravo, E., et al. 2013, A\&A, 552, A97

Isern, J., Knoedlseder, J., Jean, P., et al. 2014, ATel, 6099, 1 Jean, P., Vedrenne, G., Roques, J. P., et al. 2003, A\&A, 411, L107 Jourdain, E., \& Roques, J. P. 2009, ApJ, 704, 17

Khokhlov, A. M. 1991, A\&A, 245, 114

Kushnir, D., Katz, B., Dong, S., Livne, E., \& Fernández, R. 2013, ApJ, 778, L37 Lebrun, F., Leray, J. P., Lavocat, P., et al. 2003, A\&A, 411, L141

Lund, N., Budtz-Jørgensen, C., Westergaard, N. J., et al. 2003, A\&A, 411, L231

Marion, G. H., Sand, D. J., Hsiao, E. Y., et al. 2015, ApJ, 798, 39

Mas-Hesse, J. M., Giménez, A., Culhane, J. L., et al. 2003, A\&A, 411, L261

Matz, S. M., Share, G. H., Leising, M. D., Chupp, E. L., \& Vestrand, W. T. 1988, Nature, 331, 416

Milne, P. A., Hungerford, A. L., Fryer, C. L., et al. 2004, ApJ, 613, 1101

Nomoto, K., Thielemann, F.-K., \& Yokoi, K. 1984, ApJ, 286, 644

Pakmor, R., Kromer, M., Taubenberger, S., \& Springel, V. 2013, ApJ, 770, L8

Patat, F., Taubenberger, S., Baade, D., et al. 2014, ATel, 5830, 1

Plewa, T., Calder, A. C., \& Lamb, D. Q. 2004, ApJ, 612, L37

Reynolds, S. P., Borkowski, K. J., Hwang, U., et al. 2007, ApJ, 668, L135

Roques, J. P., Schanne, S., von Kienlin, A., et al. 2003, A\&A, 411, L91

Sazonov, S. Y., Lutovinov, A. A., \& Krivonos, R. A. 2014, Astron. Lett., 40, 65

Sturner, S. J., Shrader, C. R., Weidenspointner, G., et al. 2003, A\&A, 411, L81

Sunyaev, R., Kaniovsky, A., Efremov, V., et al. 1987, Nature, 330, 227

Tanaka, M., Mazzali, P. A., Maeda, K., \& Nomoto, K. 2006, ApJ, 645, 470

Teegarden, B. J., Barthelmy, S. D., Gehrels, N., Tueller, J., \& Leventhal, M. 1989, Nature, 339, 122

Telesco, C. M., Höflich, P., Li, D., et al. 2015, ApJ, 798, 93

The, L.-S., \& Burrows, A. 2014, ApJ, 786, 141

Ubertini, P., Lebrun, F., Di Cocco, G., et al. 2003, A\&A, 411, L131

Vancura, O., Gorenstein, P., \& Hughes, J. P. 1995, ApJ, 441, 680

Vedrenne, G., Roques, J.-P., Schönfelder, V., et al. 2003, A\&A, 411, L63

Warren, J. S., Hughes, J. P., Badenes, C., et al. 2005, ApJ, 634, 376

Weidenspointner, G., Kiener, J., Gros, M., et al. 2003, A\&A, 411, L113

Winkler, C., Courvoisier, T. J.-L., Di Cocco, G., et al. 2003, A\&A, 411, L1

Woosley, S. E., \& Weaver, T. A. 1994, ApJ, 423, 371

Zheng, W., Shivvers, I., Filippenko, A. V., et al. 2014, ApJ, 783, L24 


\section{Appendix A: Theoretical models}

The gamma-ray spectrum of SNIa depends on the total amount of ${ }^{56} \mathrm{Ni}$ and its distribution within the expanding debris, which in turn depends on the burning regime of the explosion. In the case of one-dimension models, three burning modes have been identified. Table A.1 displays the main characteristics of the models used in the present study.

Pure detonations (DETO), in which carbon is ignited in the centre of a carbon-oxygen white dwarf near the Chandrasekhar's mass and the burning propagates supersonically in such a way that the star is completely incinerated to the Fe-peak elements (Arnett 1996). This model is incompatible with all the existing observations, including those obtained by INTEGRAL in the case of SN2011fe (Isern et al. 2013) and SN 2014J (Churazov et al. 2014b). It is also representative of the most massive models computed by Fink et al. (2010).

Sub-Chandrasekhar detonations ( $\mathrm{SCH}$ ) assume white dwarfs with arbitrary masses accreting helium from a companion in such a way that when the mass of this freshly accreted envelope reaches a critical value, He ignites at the bottom and induces the explosion of the white dwarf (Woosley \& Weaver 1994). The main argument against these models has so far been the nondetection of significant amounts of ${ }^{56} \mathrm{Ni}$ and ${ }^{56} \mathrm{Co}$ moving at high velocities. The SC1F and SC3F (E. Bravo, unpublished) are SCh models equivalent to Models 1 and 3 of Fink et al. (2010).

Deflagration (DEF) models assume that the star is ignited in the central regions, and the burning front propagates subsonically through all the star in such a way that the outer layers can expand and avoid complete incineration. The prototype is the W7 model (Nomoto et al. 1984).

Delayed detonations (DDT) start as a deflagration in the centre, and when the flame reaches a density of few times $10^{7} \mathrm{~g} \mathrm{~cm}^{-3}$ it turns into a detonation. Because of the low densities, characteristic burning times are too long, matter in these layers is not completely incinerated to ${ }^{56} \mathrm{Ni}$, and only intermediate-mass elements are profusely produced during this regime, in agreement with the observations (Khokhlov 1991). The DDTe (Badenes et al. 2005) model is an example. Pulsating delayed detonations (PDD), a subtype of DDT model, assume that the burning front starts at the centre, but the flame moves so slowly that it is quenched by the expansion of the white dwarf. After reaching the maximum expansion, the star contracts and triggers the explosion (Khokhlov 1991).

The models used here are the same as in Isern et al. (2013) plus the models DDT1p4 and 3Dbball. The first one was tailored to broadly reproduce the optical light curve of SN2014J. This model is centrally ignited at a density of $2 \times 10^{9} \mathrm{~g} \mathrm{~cm}^{-3}$ and makes the transition deflagration/detonation at $1.4 \times 10^{7} \mathrm{~g} \mathrm{~cm}^{-3}$. The total mass of ${ }^{56} \mathrm{Ni}$ produced is $0.65 M_{\odot}$, the mass ejected is $1.37 M_{\odot}$, and the kinetic energy is $1.32 \times 10^{51} \mathrm{erg}$. The 3 Dbball model is essentially the DDT1p4 model plus a plume of radioactive material as depicted in Fig. 12. Figure A.1 shows the different parameters that characterize the model. Although a full set of values was explored, a reasonable choice of parameters is: mass of ${ }^{56} \mathrm{Ni}$ in the conically shaped structure $0.04-0.08 M_{\odot}$, expansion

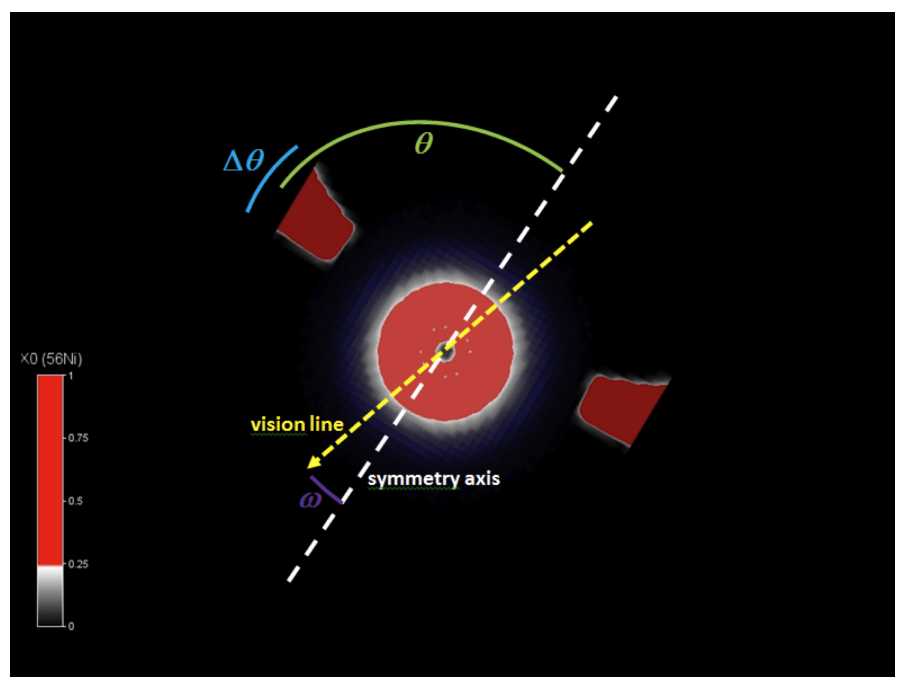

Fig. A.1. Meridional cut of a phenomenological scenario to account for the early gamma-ray emission. Two components are assumed, a central spherically symmetric remnant that contains the bulk of mass and radioactive material resulting from the explosion of the white dwarf plus a conically shaped structure made of almost pure ${ }^{56} \mathrm{Ni}$ expanding with velocities high enough to avoid being caught by the inner material.

Table A.1. Kinetic energy (K) and mass of ${ }^{56} \mathrm{Ni}$ produced by different models of explosion $\left(1\right.$ foe $\left.=10^{51} \mathrm{erg}\right)$.

\begin{tabular}{ccc}
\hline \hline Model & $K$ (foe) & $M_{\mathrm{Ni}}\left(M_{\odot}\right)$ \\
\hline DETO & 1.44 & 1.16 \\
SC3F & 1.17 & 0.69 \\
W7 & 1.24 & 0.59 \\
DDTe & 1.09 & 0.51 \\
SC1F & 1.04 & 0.43 \\
DDT1p4 & 1.32 & 0.65 \\
\hline
\end{tabular}

velocities $v_{\mathrm{i}}=25000, v_{\mathrm{s}}=35000 \mathrm{~km} \mathrm{~s}^{-1}$, while $\theta, \delta \theta$, and $\omega$ have to agree with observed recession, $\sim 6000 \mathrm{~km} \mathrm{~s}^{-1}$, and dispersion, $\sim 10000 \mathrm{~km} \mathrm{~s}^{-1}$ velocities as suggested by the redshifted Ni lines.

The gamma-ray spectrum has been obtained from a recently updated three-dimensional generalization of the code described in Gómez-Gomar et al. (1998), Milne et al. (2004), Isern et al. (2008). The initial model was obtained by adding a conical ring to the output of the DDT1p4 model as described before and allowing a homologous expansion. Given the expansion velocity that has been assumed to account for the redshift and the broadness of the line, the plume has to be clearly above the equator, $\theta \approx 78^{\circ}$, and the angular thickness $\Delta \theta \approx 12^{\circ}$. The line of sight has to be close to the axis of symmetry $\left(\omega \lesssim 12^{\circ}\right)$ since for higher values the $158 \mathrm{keV}$ line would evolve towards a doublepeaked shape that does not seem consistent with the SPI spectrum. Furthermore, the lack of substantial polarization in the optical at the early epoch also favours low values of $\omega$ (Patat et al. 2014). 